\title{
Salivary Secretory Immunoglobulin a secretion increases after 4-weeks ingestion of chlorella- derived multicomponent supplement in humans: a randomized cross over study
}

\author{
Takeshi Otsuki ${ }^{* *}$, Kazuhiro Shimizu ${ }^{2 \dagger}$, Motoyuki lemitsu $^{3 \dagger}$ and Ichiro Kono ${ }^{4 \dagger}$
}

\begin{abstract}
Background: Chlorella, a unicellular green alga that grows in fresh water, contains high levels of proteins, vitamins, minerals, and dietary fibers. Some studies have reported favorable immune function-related effects on biological secretions such as blood and breast milk in humans who have ingested a chlorella-derived multicomponent supplement. However, the effects of chlorella-derived supplement on mucosal immune functions remain unclear. The purpose of this study was to investigate whether chlorella ingestion increases the salivary secretory immunoglobulin A (SIgA) secretion in humans using a blind, randomized, crossover study design.

Methods: Fifteen men took 30 placebo and 30 chlorella tablets per day for 4 weeks separated by a 12-week washout period. Before and after each trial, saliva samples were collected from a sterile cotton ball that was chewed after overnight fasting. Salivary SlgA concentrations were measured using ELISA.

Results: Compliance rates for placebo and chlorella ingestions were $97.0 \pm 1.0 \%$ and $95.3 \pm 1.6 \%$, respectively. No difference was observed in salivary SlgA concentrations before and after placebo ingestion $(P=0.38)$. However, salivary SlgA concentrations were significantly elevated after chlorella ingestion compared to baseline $(P<0.01)$. No trial $\times$ period interaction was identified for the saliva flow rates. Although the SlgA secretion rate was not affected by placebo ingestion $(P=0.36)$, it significantly increased after 4-week chlorella ingestion than before intake $(P<0.01)$.

Conclusions: These results suggest 4-week ingestion of a chlorella-derived multicomponent supplement increases salivary SIgA secretion and possibly improves mucosal immune function in humans.
\end{abstract}

\section{Introduction}

Protein-calorie [1-3], vitamin [4-6], iron [7], and folate [8] malnutrition has been reported to cause immune deficiencies. Undernutrition is not unique to developing nations. Furthermore, in developed countries, the total food energy intake decreases with age [9] because of social [10], psychological $[11,12]$, and medical factors [13]. The nutritional status of young adults declines because of various factors such as skipping breakfast $[14,15]$, relying on fast food $[16,17]$, and dieting to achieve a thin body $[18,19]$. One of the most efficient

\footnotetext{
* Correspondence: otsuki-takeshi@rku.ac.jp

+ Contributed equally

${ }^{1}$ Faculty of Health and Sport Sciences, Ryutsu Keizai University, Ryugasaki, Ibaraki, Japan

Full list of author information is available at the end of the article
}

methods to improve the nutritional status is use of dietary supplements. However, it is not easy for the general public to choose suitable supplements for improving their individual nutritional status. For individuals with inadequate eating habits, multicomponent dietary supplements that can improve the overall immune function may be beneficial.

Chlorella, a unicellular green alga that grows in fresh water, contains high levels of proteins, chlorophylls, vitamins, minerals, and dietary fibers. This is an important advantage as an ingredient of dietary supplement. It is possible that the multicomponent dietary supplement acts to correct some underlying nutrient/nutrients deficiency and hence gets the positive outcomes such as immunoenhancing effects. Indeed, chlorella-derived
C Biomed Central

() 2011 Otsuki et al; licensee BioMed Central Ltd. This is an Open Access article distributed under the terms of the Creative Commons Attribution License (http://creativecommons.org/licenses/by/2.0), which permits unrestricted use, distribution, and reproduction in any medium, provided the original work is properly cited. 
supplements are widely used [20] and believed to enhance immune function $[21,22]$. A previous study has investigated serum antibody titers against influenza and shown the immunoenhancing effects of chlorella ingestion in middle-aged healthy adults [22]. Furthermore, supplementation with chlorella has been reported to increase the concentration of secretory immunoglobulin A (SIgA) in breast milk [21]. However, to the best of our knowledge, no studies regarding the effects of chlorella supplementation on salivary or intestinal-fluid SIgA have been reported. SIgA plays a crucial role in mucosal immune function and is the first line of defense for the human body against pathogenic microbial invasion [23]. Previous studies have reported an association between SIgA concentrations and risks of infection [24-26]. Therefore, it is of interest whether oral supplementation with chlorella can increase the salivary or intestinal-fluid SIgA secretion.

We hypothesized that ingestion of a chlorella-derived multicomponent supplement enhances mucosal immune functions. To test this hypothesis, we investigated the effects of 4-week placebo/chlorella supplementation on salivary SIgA secretion in young men using a blind, randomized, crossover study design. In the previous studies regarding the immunoenhancing effects of chlorelladerived dietary supplements, the placebo capsule was made from microcrystalline cellulose [22] or no restriction was imposed on the control group [21]. According to these studies, we attempted to make a large difference in nutritive values between the placebo and chlorella tablets based on the idea that the advantage of chlorella as a dietary supplement is to contain various nutrients.

\section{Methods}

\section{Participants}

Fifteen men volunteered to participate in this study; none of them used dietary supplements on a regular basis. The participants were asked not to change their regular lifestyles while taking the placebo/chlorella tablets during the experimental periods. None of the participants had any sign, symptom, and history of overt chronic diseases. Furthermore, none of them was taking any medication and had a history of smoking. The mean $( \pm \mathrm{SE})$ age and height values were $20.4(0.3)$ y and $1.68(0.01) \mathrm{m}$, respectively.

The present study was approved by the Ethical Committee of the Institute of Health and Sport Sciences of the University of Tsukuba. This study conformed to the principles outlined in the Helsinki Declaration. All participants gave their written informed consent before inclusion in this study.

\section{Experimental design}

The participants took part in two trials - placebo and chlorella- in a randomized order. Saliva samples were obtained from the participants after overnight fasting according to methods described in our previous studies $[27,28]$. From next day, the participants took 30 placebo or 30 chlorella (SunChlorella A; SunChlorella, Kyoto, Japan) tablets per day for 4 weeks. Saliva samples were collected again on the day following the final ingestion. Compliances with the prescription were documented via ingestion logs. After a washout period of at least 12 weeks, the second trial commenced and the procedures were same as those in the first trial.

\section{Placebo and chlorella tablets}

The mass of the placebo tablet was $243 \mathrm{mg} /$ tablet and that of the chlorella tablet was $200 \mathrm{mg} / \mathrm{tablet}$. The placebo tablets were made according to following procedures. First, a blend of lactose $(82.5 \%)$ and colorant $(17.5 \%)$ was dissolved in purified water and dried. Next, this dry matter (95\%) was mixed with sucrose fatty acid ester (5\%) and converted to tablets. The chlorella tablets contained dried chlorella pyrenoidosa powder as the main ingredient. Nutritional values of these tablets are shown in Table 1.

\section{Saliva samples}

Before the samplings, subjects refrained from alcohol consumption and intense physical activity (exercise) for $24 \mathrm{~h}$ to avoid immediate (acute) physiological effects. After overnight fasting, saliva samples were obtained in a quiet, temperature-controlled room as previously described $[27,28]$. Briefly, participants rinsed their mouths with distilled water ( $30 \mathrm{~s} \times 3$ times) and then rested for at least $5 \mathrm{~min}$. Saliva production was stimulated by chewing sterilized cotton (Salivette; Sarstedt, Nümbrecht, Germany) at a frequency of 60 chews/60 s. The amount of saliva in grams was converted to milliliters assuming a saliva density of $1 \mathrm{~g} / \mathrm{mL}$. The obtained saliva samples were separated from the cottons by centrifuging at $1,460 \mathrm{~g}$. After measurement of the sample volume, saliva samples were frozen at $-60^{\circ} \mathrm{C}$. We measured salivary SIgA concentrations using ELISA in accordance with the procedures reported in our previous studies $[27,28]$. The SIgA secretion rate $(\mu \mathrm{g} / \mathrm{min})$ was

\section{Table 1 Nutritional values of placebo and chlorella} tablets

\begin{tabular}{lcc}
\hline & Placebo & Chlorella \\
\hline Energy, kcal/100 g & 406 & 399 \\
Moisture, g/100 g & 3.2 & 5.3 \\
Protein, g/100 g & 2.0 & 60.8 \\
Lipid, g/100 g & 5.9 & 9.2 \\
Saccharide, g/100 g & 85.6 & 6.3 \\
Dietary fiber, g/100 g & 1.1 & 11.9 \\
Ash, g/100 g & 2.2 & 6.5 \\
\hline
\end{tabular}


calculated by multiplying the absolute SIgA concentration $(\mu \mathrm{g} / \mathrm{mL})$ with the saliva flow rate $(\mathrm{mL} / \mathrm{min})$.

\section{Statistical analysis}

Data are expressed as mean \pm SE. Differences in the actual periods of supplementation and compliances with the ingestion protocol between the placebo and chlorella trials were tested using an unpaired $t$-test. To compare the effects of ingestion between the placebo and chlorella trials, statistical analysis was performed using repeatedmeasures two-way ANOVA (trial $\times$ period). In the case of a significant trial $\times$ period interaction, a post hoc test (Bonferroni-Dunn) was used to identify the effect of placebo/chlorella ingestion. $P<0.05$ was accepted as significant.

\section{Results}

The periods of ingestion did not differ between the placebo $(4.2 \pm 0.1 \mathrm{wk})$ and chlorella $(4.2 \pm 0.2 \mathrm{wk})$ trials $(t=0.3, P=0.73)$. Furthermore, compliances were comparable between the placebo $(97.0 \pm 1.0 \%)$ and chlorella $(95.3 \pm 1.6 \%)$ trials $(t=0.9, P=0.36)$. Table 2 shows the body weights, lean body weights, body fat percentages, and body mass indices of participants before and after oral supplementation. No trial $\times$ period interaction was observed in these indices (body weight, $F=0.2$ and $P=0.69$; lean body weight, $F=0.2$ and $P=0.67$; body fat percentage, $F=0.0$ and $P=0.98$; body mass index, $F=0.1$ and $P=0.70$ ).

Figure 1A demonstrates salivary SIgA concentrations before and after 4-week placebo/chlorella supplementation. Trial $\times$ period interaction was identified for salivary SIgA concentrations $(F=5.9, P=0.02)$. Oral supplementation with placebo did not affect salivary SIgA concentration $(P=0.38)$, but its concentration significantly increased after chlorella ingestion compared to that before ingestion $(P<0.01)$.

As described in Figure 1B, no trial $\times$ period interaction was identified for saliva flow rates $(F=0.2, P=0.64)$.

SIgA secretion rates before and after 4-week placebo/ chlorella ingestion are shown in Figure 1C. ANOVA revealed a trial $\times$ period interaction $(F=5.3, P=0.02)$. While no difference was observed in salivary SIgA secretion rates between before and after placebo supplementation
$(P=0.36)$, it significantly increased after chlorella intake compared to baseline in multiple comparisons $(P<0.01)$.

\section{Discussion}

We investigated the effects of 4-week ingestion of a chlorella-derived multicomponent supplement on salivary SIgA secretion using a blind, randomized, crossover study design. This is a first study to evaluate the effects of chlorella-derived supplement on mucosal immune functions in humans. The salient finding of this study is that both salivary SIgA concentration and secretion rate increased after 4-week chlorella supplementation compared to baseline. Similar changes were not identified in the placebo trial. We therefore concluded that oral supplementation with a chlorella-derived multicomponent supplement enhances mucosal immune function.

Salivary SIgA secretion was used to investigate the immunoenhancing effect of ingestion of a chlorelladerived multicomponent supplement. Salivary SIgA is the first line of defense against respiratory tract infections such as pneumonia and influenza [23]. Klentrou et al. [24] reported that an increase in salivary SIgA concentration following 12 weeks of moderate exercise was related to a decrease in a number of sick days. Furthermore, Gleeson et al. [25] reported that a mean salivary SIgA concentration during a 7 -month training period of elite swimmers was associated with a number of infections contracted during this period. Therefore, we believe that salivary SIgA concentrations and secretion rates are valid indices of mucosal immune function.

Several factors are responsible for reductions in nutrients and calorie intakes. Many studies have pointed out that a diminished sense of smell and taste, increased cytokine activity, altered gastrointestinal function, and altered hormone secretion may induce anorexia even in healthy older adults $[29,30]$. Further, adverse social factors such as loneliness [10], psychological factors such as depression $[11,12]$, and medical factors such as poor dentition [13] may also cause undernutrition in older adults. In young adults, contributing factors include skipping breakfast $[14,15]$, relying on fast food $[16,17]$, and dieting to achieve a thin body $[18,19]$. In particular, university students living away from home and those who have a sedentary lifestyle are at a higher risk of poor nutrition

Table 2 Body weight, lean body weight, body fat percentage, and body mass index before and after oral supplementations with placebo/chlorella

\begin{tabular}{|c|c|c|c|c|c|}
\hline & & Body weight, kg & Lean Body Weight, kg & Body fat, $\%$ & Body Mass Index \\
\hline \multirow[t]{2}{*}{ Placebo } & Before & $65.1 \pm 2.1$ & $52.9 \pm 1.5$ & $18.3 \pm 1.6$ & $23.1 \pm 0.8$ \\
\hline & $\overline{\text { After }}$ & $65.7 \pm 2.2$ & $53.3 \pm 1.4$ & $18.3 \pm 1.7$ & $23.3 \pm 0.7$ \\
\hline \multirow[t]{2}{*}{ Chlorella } & Before & $64.7 \pm 2.3$ & $52.7 \pm 1.4$ & $17.9 \pm 1.7$ & $22.9 \pm 0.8$ \\
\hline & After & $65.1 \pm 2.3$ & $53.0 \pm 1.4$ & $17.9 \pm 1.7$ & $23.0 \pm 0.8$ \\
\hline
\end{tabular}

Values are means \pm SEM. 


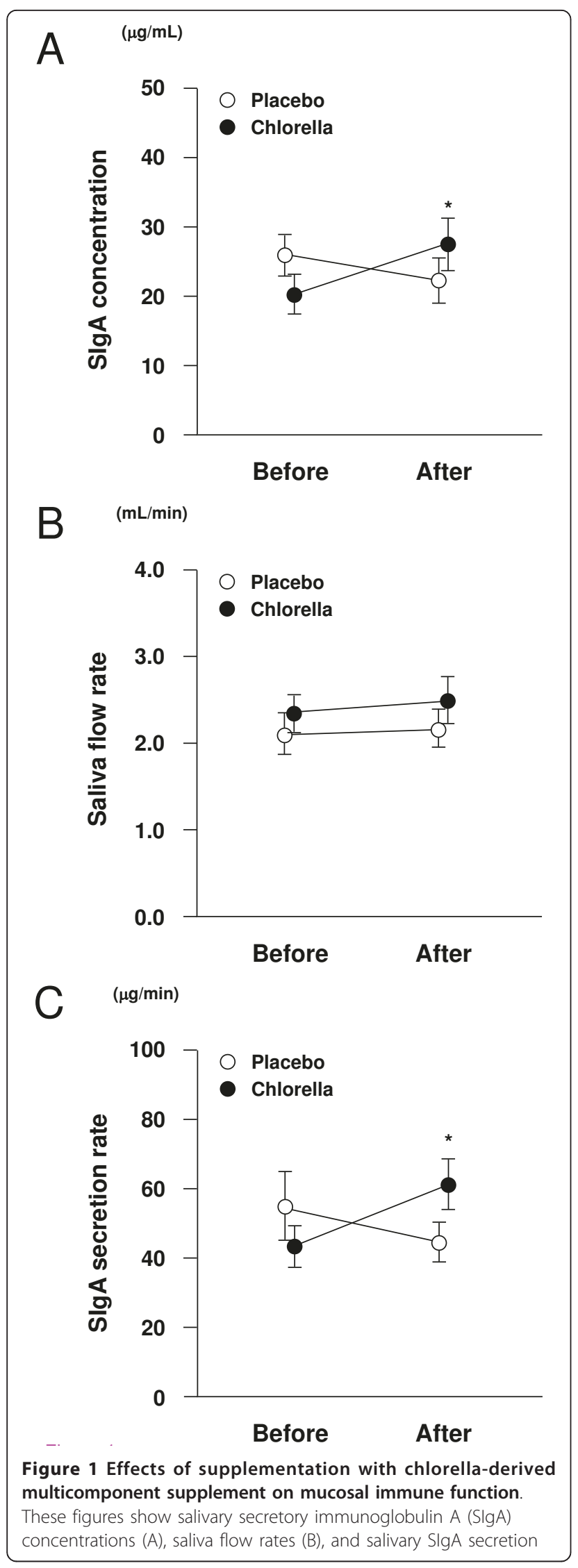

rates (C) before and after 4-week placebo $(n=15)$ /chlorella $(n=15)$ ingestion. While no difference was observed in salivary SlgA concentrations and secretion rates between before and after placebo supplementation, these indices significantly increased after chlorella intake compared to baseline. The saliva flow rates did not change after supplementation with placebo or chlorella compared to those before intervention. Data are expressed as mean \pm SE. ${ }^{*}$, significant change compared to before ingestion.

than students who live at home and participate in sporting activities [31]. The majority of the participants in this study were university students. There exists a possibility that the participants had inadequate nutritional status and the nutrients in the chlorella-derived supplement attenuated their health problems. We can speculate, from lean body weight, body fat, and body mass index, that total calorie and protein intakes in the subjects were not insufficient. However, we could not investigate their eating habits and perform any blood chemical analysis. One of the next steps is to clarify the mechanisms responsible for the chlorella ingestion-induced increase in salivary SIgA secretion.

This study has following limitations. First, we could not investigate eating habits and perform any blood chemical analysis as mentioned above. Second, we have no data describing clinical significance of change in salivary SIgA secretion. Although it is possible that the dietary supplement-related additional antibodies in healthy young humans elevate a reserve of immune function, it may not improve their infection rate at normal condition. Intervention studies in humans with reduced salivary SIgA level like athletes during training camp and older humans are needed. This study is an initial step to elucidate the effects of chlorella-derived supplement on mucosal immune functions. Recently, Yamauchi et al. [26] reported an expression of Epstein-Barr virus-DNA in saliva and an increase in a number of upper respiratory symptoms occurred on the following day of approximately 23 percent reduction in salivary SIgA secretion rate, although this reduction was smaller than the increase by the chlorella supplementation in our study (41\%). Again, the salivary SIgA secretion rate after the chlorella ingestion was 37 percent greater than that after the placebo intake. Therefore, we consider the elevation of salivary SIgA secretion rate in the chlorella trial was clinically significant. Third, we obtained saliva in the morning to equalize the conditions of subject among four sampling points (placebo or chlorella trials $\times$ before and after ingestions) although salivary SIgA level is not stable in the morning especially during $10 \mathrm{~min}$ after awakening [32]. The saliva collection in this study was performed at least 1 hour after awakening, however, we can not rule out the effect of diurnal cycle in salivary SIgA secretion. 


\section{Conclusion}

We concluded that ingestion of a chlorella-derived multicomponent supplement increases salivary SIgA secretion and possibly enhances mucosal immune function.

\author{
Author details \\ ${ }^{1}$ Faculty of Health and Sport Sciences, Ryutsu Keizai University, Ryugasaki, \\ Ibaraki, Japan. ${ }^{2}$ Sports Research \& Development Core, University of Tsukuba, \\ Tsukuba, Ibaraki, Japan. ${ }^{3}$ Faculty of sport and health science, Ritsumeikan \\ University, Kusatsu, Shiga, Japan. ${ }^{4}$ University of Tsukuba, Tsukuba, Ibaraki, \\ Japan.
}

\section{Authors' contributions}

$\mathrm{TO}$ and $\mathrm{KS}$ designed the research. TO, KS and $\mathrm{MI}$ conducted the research. TO performed the statistical analysis and wrote this paper with support from $\mathrm{KS}, \mathrm{MI}$ and IK. All authors read and approved the final manuscript.

\section{Competing interests}

SunChlorella Co., Ltd. provided funding for the study and supplied the test supplements used in the study. TO has received speaker's honorarium from SunChlorella Co., Ltd. KS, MI and IK have no competing interests.

Received: 15 February 2011 Accepted: 9 September 2011 Published: 9 September 2011

\section{References}

1. McMurray DN, Rey H, Casazza $L$, Watson RR: Effect of moderate malnutrition on concentrations of immunoglobulins and enzymes in tears and saliva of young Colombian children. Am J Clin Nutr 1977, 30:1944-1948.

2. Watson RR, McMurray DN, Martin P, Reyes MA: Effect of age, malnutrition and renutrition on free secretory component and $\lg A$ in secretions. Am J Clin Nutr 1985, 42:281-288.

3. Sirisinha S, Suskind R, Edelman R, Asvapaka C, Olson RE: Secretory and serum IgA in children with protein-calorie malnutrition. Pediatrics 1975, 55:166-170.

4. Gangopadhyay NN, Moldoveanu Z, Stephensen CB: Vitamin A deficiency has different effects on immunoglobulin $A$ production and transport during influenza A infection in BALB/C mice. J Nutr 1996, 126:2960-2967.

5. Sirisinha S, Darip MD, Moongkarndi P, Ongsakul M, Lamb AJ: Impaired local immune response in vitamin A-deficient rats. Clin Exp Immunol 1980, 40:127-135.

6. Hodges RE, Bean WB, Ohlson MA, Bleiler RE: Factors affecting human antibody response. V. Combined deficiencies of pantothenic acid and pyridoxine. Am J Clin Nutr 1962, 11:187-199.

7. Chandra RK, Saraya AK: Impaired immunocompetence associated with iron deficiency. J Pediatr 1975, 86:899-902.

8. Gross RL, Reid JV, Newberne PM, Burgess B, Marston R, Hift W: Depressed cell-mediated immunity in megaloblastic anemia due to folic acid deficiency. Am J Clin Nutr 1975, 28:225-232.

9. Centers for Disease Control and Prevention (CDC): Daily dietary fat and total food-energy intakes- Third National Health and Nutrition Examination Survey, Phase 1, 1988-91. MMWR Morb Mortal Wkly Rep 1994, 43:116-117, 123-115.

10. Walker D, Beauchene RE: The relationship of loneliness, social isolation, and physical health to dietary adequacy of independently living elderly. J Am Diet Assoc 1991, 91:300-304.

11. Wilson MM, Vaswani S, Liu D, Morley JE, Miller DK: Prevalence and causes of undernutrition in medical outpatients. Am J Med 1998, 104:56-63.

12. Smoliner $\mathrm{C}$, Norman $\mathrm{K}$, Wagner $\mathrm{KH}$, Hartig W, Lochs $\mathrm{H}$, Pirlich $\mathrm{M}$ : Malnutrition and depression in the institutionalised elderly. $\mathrm{Br} J \mathrm{Nutr}$ 2009, 102:1663-1667.

13. Sahyoun NR, Otradovec CL, Hartz SC, Jacob RA, Peters H, Russell RM, McGandy RB: Dietary intakes and biochemical indicators of nutritional status in an elderly, institutionalized population. Am J Clin Nutr 1988, 47:524-533.
14. Lien L: Is breakfast consumption related to mental distress and academic performance in adolescents? Public Health Nutr 2007, 10:422-428.

15. Tanaka M, Mizuno K, Fukuda S, Shigihara Y, Watanabe Y: Relationships between dietary habits and the prevalence of fatigue in medical students. Nutrition 2008, 24:985-989.

16. Driskell JA, Meckna BR, Scales NE: Differences exist in the eating habits of university men and women at fast-food restaurants. Nutr Res 2006, 26:524-530.

17. Morse KL, Driskell JA: Observed sex differences in fast-food consumption and nutrition self-assessments and beliefs of college students. Nutr Res 2009, 29:173-179.

18. Sano A, Le DS, Tran MH, Pham HT, Kaneda M, Murai E, Kamiyama H, Oota Y, Yamamoto S: Study on factors of body image in Japanese and Vietnamese adolescents. J Nutr Sci Vitaminol (Tokyo) 2008, 54:169-175.

19. Hayashi F, Takimoto H, Yoshita K, Yoshiike N: Perceived body size and desire for thinness of young Japanese women: a population-based survey. Br J Nutr 2006, 96:1154-1162.

20. Kay RA: Microalgae as food and supplement. Crit Rev Food Sci Nutr 1991, 30:555-573.

21. Nakano S, Takekoshi H, Nakano M: Chlorella (Chlorella pyrenoidosa) supplementation decreases dioxin and increases immunoglobulin a concentrations in breast milk. J Med Food 2007, 10:134-142.

22. Halperin SA, Smith B, Nolan C, Shay J, Kralovec J: Safety and immunoenhancing effect of a Chlorella-derived dietary supplement in healthy adults undergoing influenza vaccination: randomized, doubleblind, placebo-controlled trial. CMAJ 2003, 169:111-117.

23. Lamm ME, Nedrud JG, Kaetzel CS, Mazanec MB: IgA and mucosal defense. APMIS 1995, 103:241-246.

24. Klentrou P, Cieslak T, MacNeil M, Vintinner A, Plyley M: Effect of moderate exercise on salivary immunoglobulin $A$ and infection risk in humans. Eur J Appl Physiol 2002, 87:153-158.

25. Gleeson M, McDonald WA, Pyne DB, Cripps AW, Francis JL, Fricker PA, Clancy RL: Salivary IgA levels and infection risk in elite swimmers. Med Sci Sports Exerc 1999, 31:67-73.

26. Yamauchi R, Shimizu K, Kimura F, Takemura M, Suzuki K, Akama T, Kono I, Akimoto T: Virus activation and immune function during intense training in rugby football players. Int J Sports Med 2011, 32:393-398.

27. Akimoto T, Nakahori C, Aizawa K, Kimura F, Fukubayashi T, Kono I: Acupuncture and responses of immunologic and endocrine markers during competition. Med Sci Sports Exerc 2003, 35:1296-1302.

28. Shimizu K, Kimura F, Akimoto T, Akama T, Kuno S, Kono I: Effect of freeliving daily physical activity on salivary secretory IgA in elderly. Med Sci Sports Exerc 2007, 39:593-598.

29. Chapman IM: Nutritional disorders in the elderly. Med Clin North Am 2006 90:887-907.

30. Morley JE: Anorexia of aging: physiologic and pathologic. Am J Clin Nutr 1997, 66:760-773.

31. Shimbo S, Zhang ZW, Matsuda-Inoguchi N, Higashikawa K, Nakatsuka $H_{\text {, }}$ Watanabe T, Ikeda M: Effects of life away from home and physical exercise on nutrient intake and blood/serum parameters among girl students in Japan. Tohoku J Exp Med 2004, 203:275-286.

32. Hucklebridge F, Clow A, Evans P: The relationship between salivary secretory immunoglobulin A and cortisol: neuroendocrine response to awakening and the diurnal cycle. Int J Psychophysiol 1998, 31:69-76.

doi:10.1186/1475-2891-10-91

Cite this article as: Otsuki et al: Salivary Secretory Immunoglobulin a secretion increases after 4-weeks ingestion of chlorella-derived multicomponent supplement in humans: a randomized cross over study. Nutrition Journal 2011 10:91. 